\title{
Kinderonkologische Intensivmedizin
}

\author{
Arndt Borkhardt
}

\section{Übersicht}

Einleitung

Erkrankungsassoziierte

Notfallsituationen

Therapiebedingte Notfälle

\section{Einleitung}

Vor dem Hintergrund der mittlerweile guten bis sehr guten, teils sogar exzellenten Prognose vieler onkologischer Erkrankungen im Kindesalter ist das erfolgreiche Vorgehen in typischen Notfallsituationen von besonders hoher Relevanz. Die häufigsten Gründe für die Aufnahme auf die Intensivstation sind ein infektionsbedingtes respiratorisches Versagen oder eine volumenrefraktäre Hypotonie in Folge eines septischen Schocks. Ein primär kardiales Versagen kommt seltener vor, kann jedoch insbesondere bei einem malignen Perikarderguss (cave: Burkitt-Lymphom) oder nach hoher kumulativer Anthrazyklindosis ebenfalls vorkommen.

Ein intensivmedizinischer Interventionsbedarf bei Kindern mit maligner Systemerkrankung oder solidem Tumor kann durch die Grunderkrankung oder durch die Nebenwirkungen einer medikamentösen Therapie entstehen.

\section{Erkrankungsassoziierte Notfallsituationen}

\section{Thymus- bzw. Mediastinaltumor}

T-Zell-Neoplasien zeichnen sich durch eine hohe intrinsische Proliferationsrate aus. Kinder mit T-Zell-Leukämie, T-Zell-Non-Hodgkin-Lymphom (Abb. 1), selte- ner auch Keimzelltumor oder Neuroblastom, können deshalb rasch eine mechanisch bedingte respiratorische Insuffizienz durch einen Thymustumor entwickeln.

Kinder mit Mediastinaltumor können oft schon eine ganz erhebliche kompressionsbedingte Lumenminderung der Atemwege aufweisen, ohne dass deutliche klinische Zeichen auf die drohende schwere Respirationsstörung hinweisen.

Ein Dilemma ergibt sich häufig daraus, dass für die weitere Therapie der Grunderkrankung die genaue morphologische, zyto- und molekulargenetische Charakteristik der Raumforderung im vorderen oberen Mediastinum entscheidend ist, was eine Biopsie notwendig

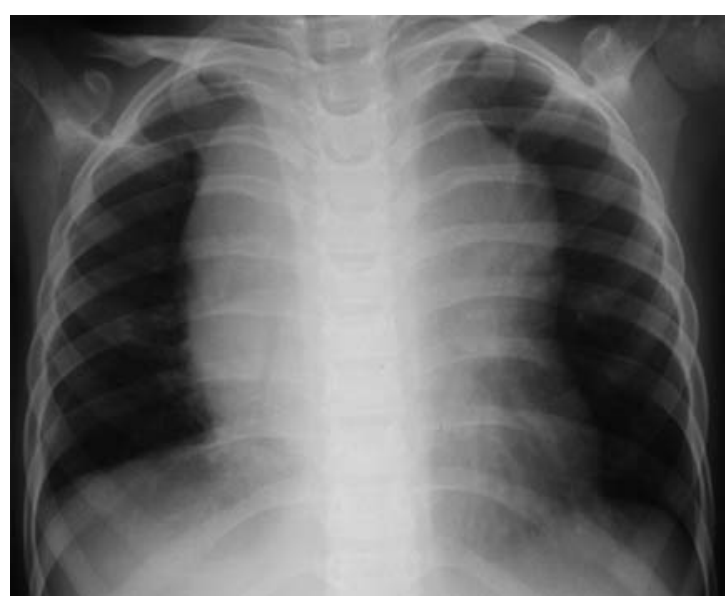

Abb. 1 Typisches Bild eines T-ZellNon-HodgkinLymphoms. 
macht. Andererseits drohen aber selbst bei kleineren invasiven Eingriffen, also auch bei einer Biopsie, schwere Narkosekomplikationen im Sinne eines „mediastinal mass syndrome“. Wenn das Blutbild in Verbindung mit erhöhten LDH- und Harnsäurewerten Hinweise auf eine Leukämie bietet, ist daher eine Knochenmarkpunktion in Lokalanästhesie und milder Sedierung einer Biopsie bei Weitem vorzuziehen. Einen begleitenden Pleuraoder Perikarderguss punktiert man vorab, da bereits das Zytozentrifugenpräparat oft eine definitive Diagnose erlaubt und sich außerdem durch die Punktion die Symptomatik rasch bessern kann.

Differenzialdiagnostisch können mediastinale Keimzelltumoren aufgrund oft exorbitant erhöhter Tumormarker ( $\beta$-HCG, $\alpha$-1-Fetoprotein) und mediastinale Neuroblastome anhand der Katecholaminabbauprodukte (Homovanillinmandelsäure, Vanillinmandelsäure) abgegrenzt werden. Die Metaboliten eines in ca. $80 \%$ katecholaminproduzierenden Neuroblastoms führen intermittierend oft zu stark hypertonen Blutdruckwerten

Wenn die primäre invasive Diagnostik eines großen Mediastinaltumors wegen eines lebensbedrohlichen Zustandsbilds unmöglich ist, ist empirische zytoreduktive Therapie mit Prednison (0,5-1 mg/ $\mathrm{kg} \mathrm{KG)} \mathrm{notwendig.} \mathrm{Bei} \mathrm{ausbleibendem} \mathrm{Therapie-}$ erfolg (Zeichen der Tumorlyse im Blut? Abnahme der Raumforderung?) ist die Therapie schon nach $24-48$ h mit Cyclophosphamid (100-200 mg/m² Körperoberfläche) zu erweitern.

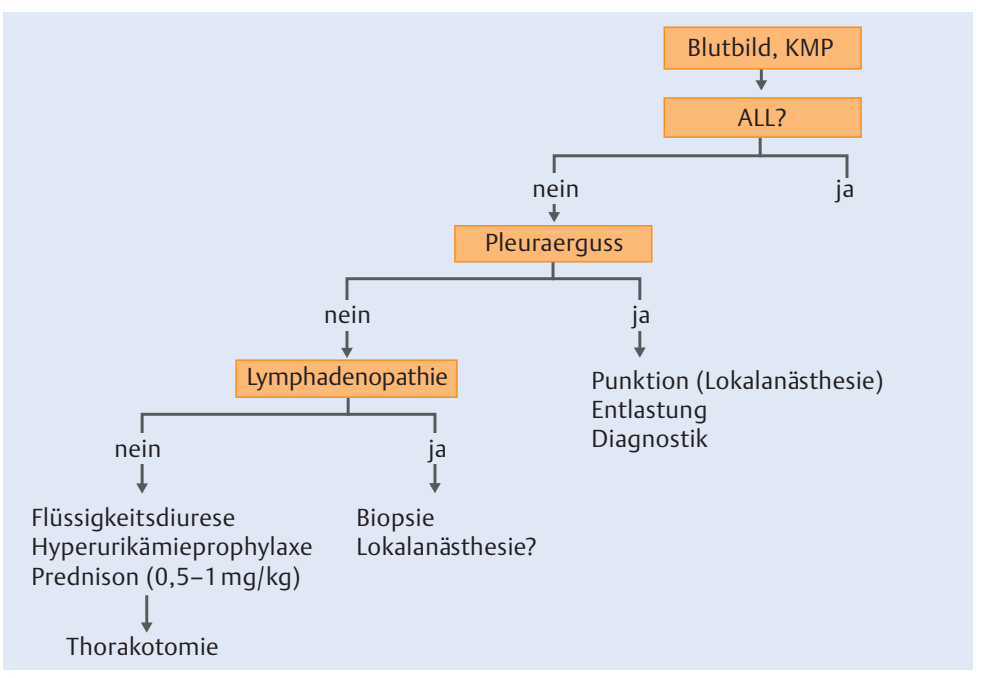

Abb. 2 Algorithmus bei Mediastinaltumor ohne respitaorische Beeinträchtigung.

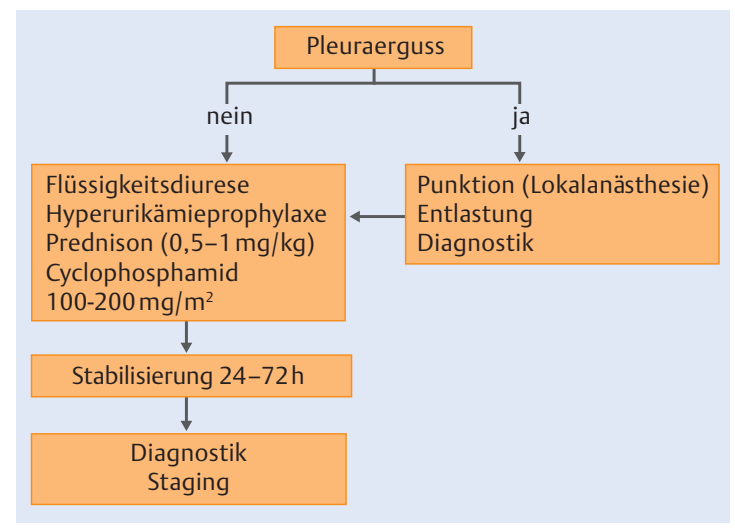

Abb. 3 Algorithmus bei Mediastinaltumor mit Orthopnoe.

\section{Infobox 1}

Onkologische Ursachen einer Rückenmarkkompression im Kindesalter

- Ewing-Sarkom

- Osteosarkom

- Weichteilsarkome

- Neuroblastome

- Lymphome

- ZNS-Tumoren (Ependymome)

- Blutungen

Klinische Zeichen:

- Schmerzen

- motorische und/oder sensorische Ausfälle

- Blasen-/Mastdarmdysfunktion

Nach 48-72 h und in der Regel rasch schrumpfender Raumforderung kann man die Biopsieentnahme meist gefahrlos durchführen. Das Vorgehen bei Mediastinaltumoren ist in Abb. 2 und $\mathbf{3}$ zusammengefasst.

Wegen der oft hohen Chemotherapie-Sensitivität von Non-Hodgkin-Lymphomen, Leukämien und Keimzelltumoren sollte man allerdings keine ungezielte, empirische Therapie mehr einsetzen, da sonst möglicherweise eine genaue histologische Befunderstellung unmöglich wird.

\section{Rückenmarkkompression}

Die onkologischen Ursachen einer Rückenmarkkompression im Kindesalter sind zahlreich (Infobox 1). Die Symptomatik beginnt oft unspezifisch mit Kopf- oder Rückenschmerzen. 
Bei Kindern mit maligner Erkrankung und Kreuzschmerzen besteht solange der Verdacht auf eine Rückenmarkkompression, bis das Gegenteil bewiesen ist.

Bei Non-Hodgkin-Lymphomen ist das Rückenmark in der Regel nicht der einzige Befallsort. Die Wahrscheinlichkeit einer irreversiblen neurologischen Schädigung hängt ab vom Ausmaß, v.a. aber auch von der Dauer der Rückenmarkkompression.

Eine Myelonkompression erfordert rasches Handeln. Das Intervall zwischen ersten neurologischen Symptomen und einem kompletten Querschnitt ist oft sehr kurz (bei Non-Hodgkin-Lymphomen in der Regel weniger als 1 Woche).

Wenn die Diagnose einer lymphatischen Neoplasie aus anderen Manifestationsorten gesichert werden kann, ist eine unverzügliche Therapie mit hochdosierten Steroiden einzuleiten. In allen anderen Fällen ist die rasche operative Dekompression unumgänglich (meist durch Hemilaminektomie, seltener komplette Laminektomie oder nur durch Laminotomie). Die neurologische Restitution hängt jedoch nicht von einer kompletten Resektion des Tumors ab.

\section{Hyperleukozytose}

Ungefähr jedes 10. Kind mit akuter lymphatischer oder akuter myeloischer Leukämie weist bei Diagnosestellung Leukozytenzahlen über $100000 \mu$ auf. Durch die Hyperleukozytose kann es zur Stase in Lungen- und Hirngefäßen kommen.

Die Gefäßwandhypoxie und die stark erniedrigte Blutviskosität bedingen ein hohes Risiko für teils massive Blutungen.

AML. Vorläuferzellen der Myelopoese wie Myeloblasten und Monoblasten sind im Vergleich zu Lymphoblasten oder reifen Granulozyten wenig verformbar. Dies ist der Grund für die besondere Blutungsgefahr bei akuten Monoblastenleukämien (AML FAB-M5).

Eine Besonderheit ist die sehr seltene akute Promyelozytenleukämie (AML FAB-M3). Die Kinder haben eine sehr gute Aussicht auf Heilung, sofern sie komplikationsfrei eine erste Remission erreichen. In den ersten Therapietagen können bei dieser Leukämieform jedoch selbst bei sonst ungefährlichen Leukozytenwerten von nur 20000/ $\mu$ l schwere Blutungen auftreten.

\section{Kasuistik}

\section{Rückenmarkkompression}

Bei einem vorher gesunden 6-jährigen Jungen kommt es seit 6 Wochen zu rezidivierenden, teils auch heftigen Attacken mit Kopfschmerz und Nasenbluten. Eine ambulante HNO-ärztliche und kinderärztliche Vorstellung bleibt bis auf eine leichte Anämie ohne wegweisenden Befund. Eine plasmatische oder thrombozytäre Blutungsneigung wird ausgeschlossen.

Eines Abends klagt der Junge über Bauch- und Kreuzschmerzen sowie Schmerzen in beiden Beinen. Am nächsten Morgen fällt den Eltern eine deutliche Gangunsicherheit auf. Das Kind wird in die Kinderklinik eingewiesen, wo eine spinale MRT den hochgradigen Verdacht auf eine langstreckige paraspinale Raumforderung mit Einwachsen in die Foramina intervertebralia zeigt. Umgehend wird eine Hemilaminektomie zur Dekompression des Rückenmarks durchgeführt. Histologisch wird die Verdachtsdiagnose eines Neuroblastoms gesichert (Abb. 4).
Klinisch geht die Hyperleukozytose oft mit einer erheblichen Tachydyspnoe und Zyanose einher. Man findet diffuse infiltrative Veränderungen in den Lungen. Doppelbilder, Nystagmus, Ataxiezeichen und gelegentlich ein sehr schmerzhafter Priapismus können vorkommen.

Die Indikationen zur Austauschtransfusion ergibt sich aus der Zusammenschau von klinischem Bild, vorliegendem Subtyp der Leukämie und der Leukozytenzahl

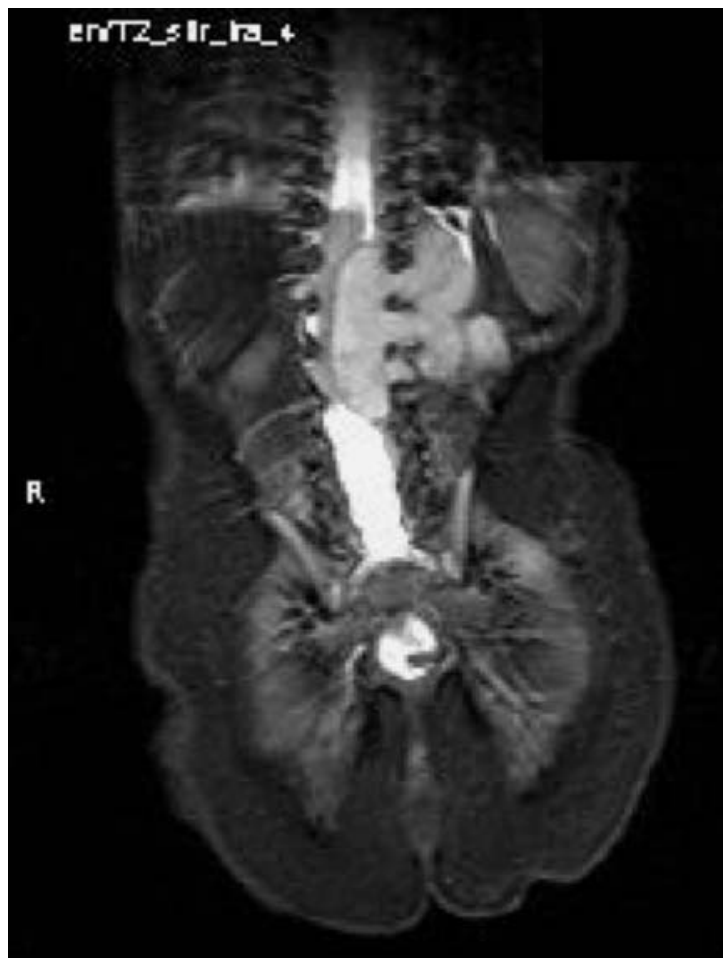

Abb. 4 Typische sanduhrförmige Tumorformationen eines Neuroblastoms. 
im peripheren Blut. Als Faustregel kann gelten, dass die

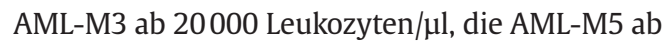
100000 Leukozyten/ $\mu$ l und andere AML-Formen ab

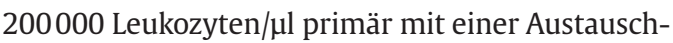
transfusion behandelt werden sollten.

Insbesondere bei jungen Säuglingen mit AML (meist Monoblastenleukämien) besteht das erhebliche Risiko einer intrazerebralen Blutung auch nach erfolgreicher Austauschtransfusion noch für 4- 5 Tage fort. Die Praxis zeigt, dass eine umfangreiche Substitution von Gerinnungsfaktoren bei diesen Kindern oft zu früh vernachlässigt wird.

ALL und CML. Bei akuten lymphoblastischen Leukämien (ALL) und der im Kindesalter seltenen chronischmyeloischen Leukämie (CML) sollte sich die Entscheidung zur primären Austauschtransfusion primär am klinischen Bild und weniger an der Leukozytenzahl orientieren. Wichtig ist auch der dynamische Verlauf der Leukozytenzahl unter der spezifischen antileukämischen Induktionstherapie. Bei einem unzureichenden Leukozytenabfall muss die Entscheidung zur antileukämischen Therapieintensivierung täglich neu überdacht werden oder ggf. auch sekundär eine Austauschtransfusion erwogen werden.

\section{Hirntumoren, Hirndruck}

Tumoren des Zentralnervensystems sind die häufigsten soliden Neoplasien im Kindesalter. Während sich Hirntumoren bei Erwachsenen hauptsächlich in den Hemi- sphären entwickeln, befinden sie sich bei Kindern zu fast zwei Dritteln infratentoriell.

Symptomatik. Tumoren, die in der hinteren Schädelgrube oder in der Nähe des III. Ventrikels angesiedelt sind, führen relativ früh zu einem Liquorstau. Die Erhöhung des intrakraniellen Drucks bedingt eine $\mathrm{Ab}$ nahme des zerebralen Perfusionsdrucks. Diesen berechnet man näherungsweise, indem man vom mittleren arteriellen Blutdruck den intrakraniellen Druck abzieht.

Therapie. Bei Kindern mit Hirntumor und Symptomen einer intrakraniellen Hypertension ist die Liquordrainage die Therapie der Wahl. Sie ist eine einfache und effektive Maßnahme, wenn nicht primär eine vollständige Tumorresektion angestrebt werden kann. Die im klinischen Alltag immer wieder neu zu diskutierende Frage nach der Art der Ableitung (extern oder intern, mit oder ohne Tumorfilter, simultane Implantation eines Rickham-Reservoirs für die postoperative Chemotherapie) hängt neben Tumorsitz, vermuteter Tumorart und Liquorzusammensetzung auch davon ab, ob nach einer anschließenden Tumorresektion voraussichtlich eine Liquorzirkulationsstörung bestehen bleiben wird oder nicht.

Der größte Nachteil einer passageren externen Ableitung ist die große Infektionsgefahr. Deshalb sollte man alle $24 \mathrm{~h}$ kritisch hinterfragen, ob die externe Liquorableitung noch den klinischen Erfordernissen entspricht. Durch Chemotherapie ausgelöste Neutropeniephasen erhöhen das Infektionsrisiko überproportional.

\section{Therapiebedingte Notfälle}

\section{Tumorlysesyndrom}

In der Kinderonkologie kommt ein Tumorlysesyndrom hauptsächlich bei 4 Erkrankungen vor:

- Burkitt-Lymphom (B-ALL),

- akute lymphoblastische Leukämie vom T-Zell-Typ,

- akute myeloische Leukämie,

- Säuglingsleukämien (hohe initiale Leukämiezellzahl).

Ein Zerfall von Tumorzellen - insbesondere von Leukämie- und Lymphomzellen - überschwemmt den Körper mit Elektrolyten (Kalium, Phosphat; Tab. 1) sowie DNA-Abbauprodukten wie Xanthin, Hypoxanthin und 
Harnsäure. Bei Überschreiten des Löslichkeitsprodukts kristallisieren diese in den Nierentubuli und Sammelrohren aus. Ausfallendes Kalziumphosphat findet sich auch in der peripheren Endstrombahn (Pankreas!).

Ein metabolisches Ungleichgewicht droht immer dann, wenn es nicht gelingt, einen ausreichenden Urinfluss $\left(200 \mathrm{ml} / \mathrm{m}^{2} / \mathrm{h}\right.$ ) aufrechtzuerhalten. Eine Urinalkalisierung verringert die Wahrscheinlichkeit einer Kristallisation von Xanthin und Harnsäure, begünstigt aber die Ausfällung von Kalziumphosphat. Ob eine zusätzliche Bikarbonatgabe zur Prävention des Zellzerfallsyndroms hilfreich sein kann, ist also im Einzelfall abzuwägen.

Die Hyperkaliämie ist die häufigste unmittelbar lebensbedrohliche Komplikation eines akuten Zellzerfallsyndroms.

Beim Einleiten einer chemotherapeutischen Behandlung ist die Monitorüberwachung daher obligat.

Als Pseudohyperkaliämie wird eine Kaliumerhöhung erst nach der Blutentnahme durch einen fortgesetzten Zellzerfall ex vivo bezeichnet. Sie tritt insbesondere bei anfänglich hoher Leukämiezellzahl im peripheren Blut auf. Eine Blutabnahme bei zu langer Stauung kann durch „Quetschkalium“ ebenfalls falsch hohe Werte vortäuschen.

Zur Notfall-Therapie der Hyperkaliämie und Hyperurikämie s. Infobox 2.

\section{Thrombosen, speziell Sinusvenenthrombose}

Die wachsende Zahl neu entdeckter, potenziell wichtiger hereditärer Thrombophiliefaktoren erschwert es, den Überblick zu behalten. Zudem ist die Studienlage zum prädiktiven Wert einzelner Befundkonstellationen oft widersprüchlich. Daher gibt es keinen allgemeingültigen Konsens, welche Risikofaktoren bei welcher onkologischen Grunderkrankung vor Therapiebeginn bestimmt werden sollten.

Die Kinder bekommen heute praktisch immer einen permanenten zentralvenösen Katheter zur sicheren Applikation der Chemotherapie implantiert. Einige Chemotherapeutika - insbesondere die L-Asparaginase - sind zudem mit einem deutlich erhöhten thrombogenen Risiko assoziiert. Die Therapie mit L-Asparaginase führt zu einem Ungleichgewicht pro- und antiko-

\section{Infobox 2}

Therapie der Hyperkaliämie und Hyperurikämie
Notfall-Behandlung der Hyperkaliämie mit QT-Verbreiterungen und/oder hohen T-Wellen:

- Kalziumgluconat 10\%: 0,25$0,5 \mathrm{ml} / \mathrm{kg}$ KG über $10 \mathrm{~min}$ i.v.,

- EKG-Überwachung, cave Bradykardie.

Wenn noch keine EKG-Veränderungen sichtbar sind:

- Salbutamolinhalation: 0,5-1 ml Inhalationslösung auf $3 \mathrm{ml}$ verdünnt,

- Natriumbikarbonat: 2 mmol/ kg KG verdünnt mit Aqua dest.,

- Glukose-/Insulintropf.
Behandlung der Hyperurikämie:

- Allopurinol ist sicher und effektiv (als Nebenwirkung allergische Hautreaktionen möglich),

- rekombinante Uratoxidase spaltet Harnsäure sehr effektiv: $0,15 \mathrm{mg} / \mathrm{kg}$ KG als Kurzinfusion.

Cave: Bei Therapie mit rekombinanter Uratoxidase Blutprobe nach der Entnahme sofort auf Eis legen, sonst führt die fortgesetzte Enzymaktivität während des Probentransports zu einem Harnsäurewert von Null. agulatorischer Faktoren. Zudem wird das für die erfolgreiche Behandlung der akuten lymphoblastischen Leukämie (ALL) äußerst wichtige Medikament oft mit Glukokortikoiden kombiniert, was das Thromboserisiko zusätzlich erhöht. Die Rolle des unter der Chemotherapie einer ALL oft stark erniedrigten Fibrinogens für das Thromboserisiko ist unklar.

Bei subnormalen Werten für AT III oder Firbrinogen kann eine großzügige Substitution von AT III oder FFP nicht empfohlen werden.

Symptomatik. Die recht häufige Sinusvenenthrombose hat insgesamt eine gute Prognose. Die Kinder fallen mit starken Kopfschmerzen, dem klinischen Bild eines Pseudotumor cerebri, Aphasien oder Sehfeldausfällen auf. Lang anhaltende Krampfanfälle sind dagegen selten.

Therapie. Eine aggressive Lysetherapie einer radiologisch gesicherten Sinusvenenthrombose ist wegen der hohen Blutungsgefahr kontraindiziert. In der Regel ist die Therapie mit niedermolekularem Heparin (z.B. $2 \times 1 \mathrm{mg} / \mathrm{kg} \mathrm{KG/d}$, abhängig vom Präparat) sicher und effektiv. Eine weitere Apposition von thrombotischem Material wird damit verhindert, die (partielle) Re-Kanalisierungsrate ist hoch. Der 4-h-Anti-Xa-Spiegel sollte bei einer nachgewiesenen Sinusvenenthrombose zwischen 0,7 und $1 \mathrm{U} / \mathrm{ml}$ liegen. 
Abb. 5

Diagnostisches Vorgehen bei Fieber und Neutropenie.

Fieber und Neutropenie

Anamnese, körperliche Untersuchung

Blutbild, Gerinnung, CRP, Leber- und Nierenfunktionsparameter Blutkultur: Bakterien, Pilze, (peripher, ggf. alle Katheterschenkel) Urinkultur: Bakterien, Pilze, Viren

Nasen-Rachen-Abstrich: Bakterien, Pilze, Viren Aspergillus- und Candida-Antigen

Bei Fokus/Symptomatik weiterführende Diagnostik (z.B. Stuhl auf Bakterien, Viren, Pilze Clostridium difficile - Toxin bei Diarrhoe, Sputum und Röntgenthorax bei Husten
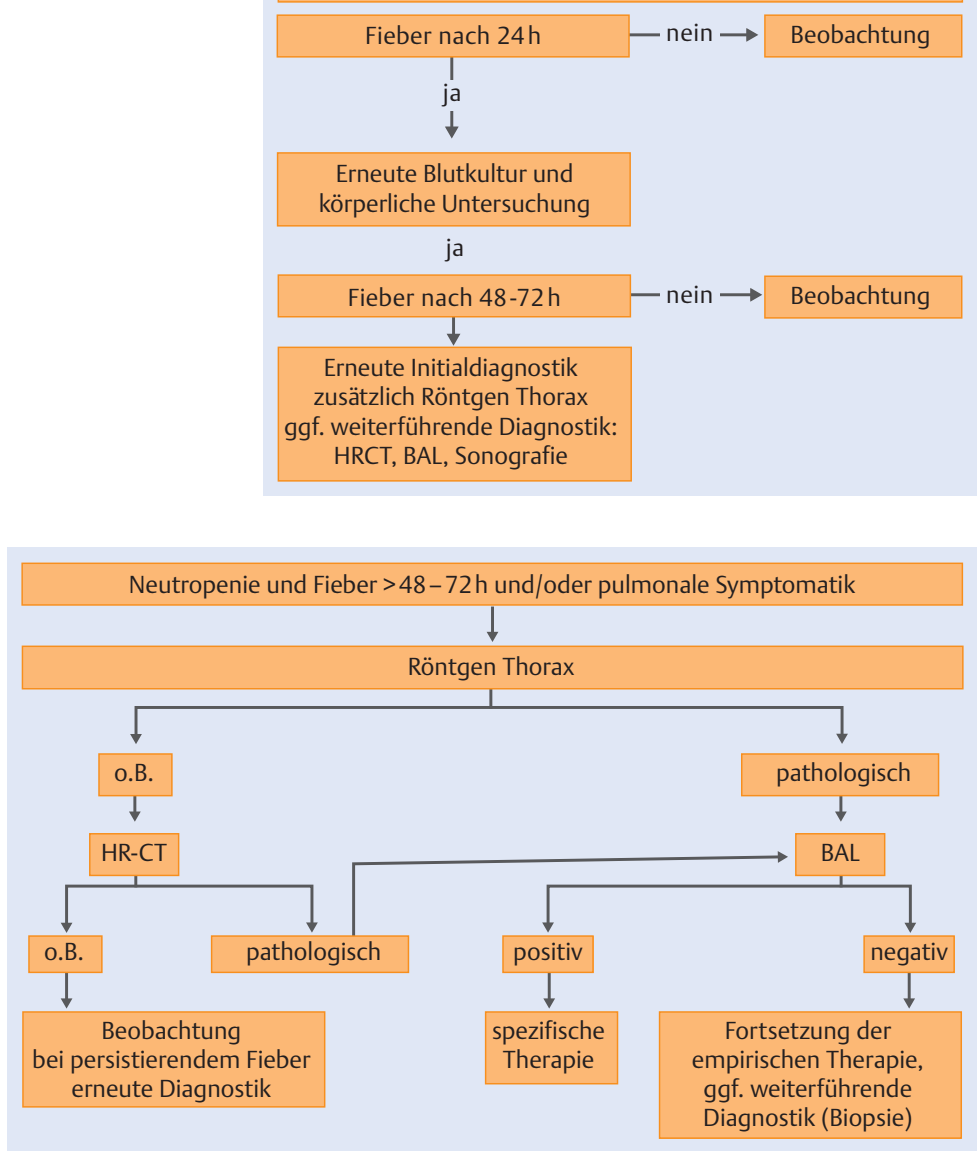

Abb. 6 Vorgehen bei Fieber und Neutropenie länger als 48-72 Stunden.

\section{Neutropenisches Fieber}

Symptomatik. Fieber ist häufig das einzige Symptom bei einem Kind in der durch die Chemotherapie induzierten Neutropeniephase. Als Fieber wird eine einmalige Körpertemperatur von über $38,5^{\circ} \mathrm{C}$ oder eine im Abstand von $4 \mathrm{~h}$ zweimal gemessene Temperatur von über $38^{\circ} \mathrm{C}$ bezeichnet. Bei $30-40 \%$ der Patienten gelingt der Erregernachweis aus der Blutkultur oder einem lokalen Wundabstrich (Kathetereintrittstelle). Bei einem Großteil der Kinder jedoch bleibt es bei der Diagnose „unklares Fieber“ („fever of unknown origin“, FUO).
Therapie. Eine Strategie zur Therapie eines Fiebers bei Neutropenie ist in Abb. 5 und $\mathbf{6}$ zusammengefasst.

Die unverzüglich einzuleitende empirische antibiotische Behandlung muss sich nach dem lokalen Keimspektrum, lokalen Resistenzproblemen und nicht zuletzt nach der eigenen pädiatrisch-onkologischen $\mathrm{Pa}-$ tientengruppe mit ihrer spezifischen Chemotherapieintensität richten.

Ein Beispiel für ein therapeutisches Vorgehen bei Kindern mit unklarem Fieber ist in (Tab. 2) zusammengefasst.

\section{Methotrexat-Ausscheidungsstörung}

Methotrexat (MTX) ist ein Strukturanalogon der Folsäure. Es wird seit Jahrzehnten mit Erfolg zur Behandlung von akuten lymphatischen Leukämien und Osteosarkomen in der Kinderonkologie eingesetzt. Grundlage der antineoplastischen Wirkung sind intrazelluläre MTX-Polyglutamate, die ihrerseits Enzyme im Syntheseprozess der Nukleosidvorstufen und der de-novo Purinsynthese hemmen. Ohne eine zeitlich versetzte Antagonisierung und den Schutz normaler Körperzellen mit dem Antidot Leukovorin (überbrückt den MTX-induzierten Dehydrofolatreduktaseblock) ist eine i.v. Gabe größerer Mengen MTX immer letal. Neben regelmäßigen Messungen des MTX-Plasmaspiegels ist auf eine ausreichende Hydrierung und Alkalisierung (Urin pH 7,5) zu achten.

Symptomatik. Die Nebenwirkungen umfassen neben einer oft erheblichen Schleimhaut- und Hämatotoxizität auch eine plötzliche Einschränkung der Nierenfunktion. Es kann zur Präzipitation von MTX und seinen Metaboliten in den Nierentubuli kommen. Da MTX fast ausschließlich über die Niere ausgeschieden wird, kann sich hieraus schnell ein sehr bedrohliches Krankheitsbild entwickeln. Schäden an Knochenmark und Leber sowie die akute Entwicklung einer schweren Enzephalopathie mit Krampfanfällen werden regelmäßig beobachtet.

Unter einer hochdosierten ( 5 - $12 \mathrm{~g} / \mathrm{kg} \mathrm{KG}$ ) MTX-

Therapie sind neben dem MTX-Plasmaspiegel auch die Nierenfunktionsparameter eng zu überwachen. Auch eine diskrete Kreatinin- oder Harnstofferhö-

hung kann ein ernstes Warnsignal sein. 
Therapie. Die ersten Maßnahmen bei einem erhöhten MTX-Plasmaspiegel umfassen neben einer weiteren Steigerung der Flüssigkeitszufuhr $\left(5-6 \mathrm{l} / \mathrm{m}^{2}\right)$ selbstverständlich auch die wiederholte Gabe einer erhöhten Dosis des Antidots Leucovorin. Die konkrete Leucovorindosis richtet sich nach der zuvor applizierten MTX-Dosis, dem Infusionsintervall und dem aktuell gemessenen MTX-Plasmaspiegel.

In diesen Situationen vermeidet man grundsätzlich die Gabe von Medikamenten, die den Folatmetabolismus hemmen (z. B. Trimethoprim-Sulfametoxazol) oder die Einweißbindung senken.

Je nach Schweregrad der verzögerten MTX-Ausscheidung (6-stündige Spiegelmessungen) ist zunächst eine adäquate Erhöhung des „LeucovorinRescues“ erforderlich. Bei zu schneller i. v. Gabe von Leucovorin sind aber aufgrund des Kalziumgehalts der Injektionslösung kardiale Rhythmusstörungen möglich.

Sollte eine verlängerte und erhöhte Leucovorin-Rescue nicht ausreichen, so setzt man das rekombinante Enzym Glucarpidase bzw. Carboxypeptidase G2 (50 U/ $\mathrm{kg} \mathrm{KG)} \mathrm{zur} \mathrm{schnellen} \mathrm{und} \mathrm{effektiven} \mathrm{Spaltung} \mathrm{des} \mathrm{MTX}$ (>99\%) ein. Da es sich um ein hochwirksames, aber bisher in der EU nicht zugelassenes und teures Medikament handelt ( 2 Ampullen à 1000 U zur Behandlung eines ca. $40 \mathrm{~kg}$ schweren Patienten kosten rund $14000 €$ ), wird Glucarpidase in der Praxis eher zu spät als zu früh eingesetzt. Oft sind zu diesem Zeitpunkt leider schon irreversible Organschäden eingetreten. Als Faustregel kann gelten, dass bei einem protrahierten MTX-Plasmaspiegel über $10 \mu \mathrm{mol} / 1$ die Indikation gegeben ist. Das Medikament kann über eine rund um die Uhr besetzte Notfallnummer (0044-1932-8241-00 oder-98) direkt aus Großbritannien bezogen werden.

\section{Nierenersatzverfahren zur Entfernung von MTX und} seiner toxischen Metaboliten sind weniger wirksam.

Auch nach der Gabe von Glucarpidase ist die Hydrierung und Alkalisierung unbedingt fortzuführen, da das entstehende MTX-Abbauprodukt DAMPA ebenfalls schlecht wasserlöslich ist.

Nach enzymatischer MTX-Spaltung sind StandardAssays zur Messung des verbliebenen MTX-Plasmaspiegels ungeeignet. Sie liefern falsch hohe Werte. Eine HPLC-gestützte MTX-Spiegelmessung ist deshalb anzustreben.

\section{Tabelle 2}

Beispiel für das therapeutische Vorgehen bei Kindern mit unklarem Fieber.

anfängliche Therapie bei Fieber über $38,5^{\circ} \mathrm{C}$

Ceftazidim (100 mg/kg KG/d in 2 Dosen)

plus

Tobramycin $(5 \mathrm{mg} / \mathrm{kg} / \mathrm{KG} / \mathrm{d}$ i. v. in 2 Dosen)

bei Nichtentfieberung nach $72-96 \mathrm{~h}$ zusätzlich

bei Nichtentfieberung nach weiteren 24-48 h zusätzlich

\section{Paravasate, Fehlapplikationen}

Die Wirkung von Paravasaten aus peripheren Venen ist je nach Chemotherapeutikum sehr unterschiedlich. Stark nekrotisierend sind Actinomycin D, Amasacrine, die Substanzgruppe der Anthrazykline und insbesondere Vincristin und Vindesine.

Vinca-Alkaloide. Vincristin und Vindesine sollte man sofern sie nicht ohnehin über einen zentralvenösen Katheter verabreicht werden - immer durch direkte Punktion der Vene oder einen neu angelegten i.v. Zugang applizieren. Injektionen über dem Handgelenk oder dem Ellenbogengelenk muss man möglichst vermeiden.

Eine immer wieder vorkommende Verwechslung betrifft die fatale intrathekale Applikation von Vinca-Alkaloiden. Dabei kommt es fast regelhaft zu einer aufsteigenden degenerativen Radikulomyeloenzephalopathie, die meist nach Tagen oder Monaten den Tod zur Folge hat. In einigen Chemotherapieprotokollen wurde deswegen strikt darauf geachtet, dass intrathekal zu applizierende Therapieelemente grundsätzlich nicht an Tagen vorgesehen sind, an denen eine i.v. Gabe von Vinca-Alkaloiden geplant ist.

Obwohl die Erstbeschreibung dieser Medikamentenverwechslung fast 50 Jahre zurück liegt, erfasst auch die aktuellere Literatur immer wieder ähnlich gelagerte tragische Fälle. Die therapeutischen Möglichkeiten sind sehr begrenzt, auch wenn es mitunter Wochen dauert, bis die aufsteigende Paralyse langsam zum Tod führt. Ob die vereinzelt überlebenden Patienten tatsächlich von einem unverzüglichen Liquoraustausch und neurochirurgischen Interventionen profitiert oder 


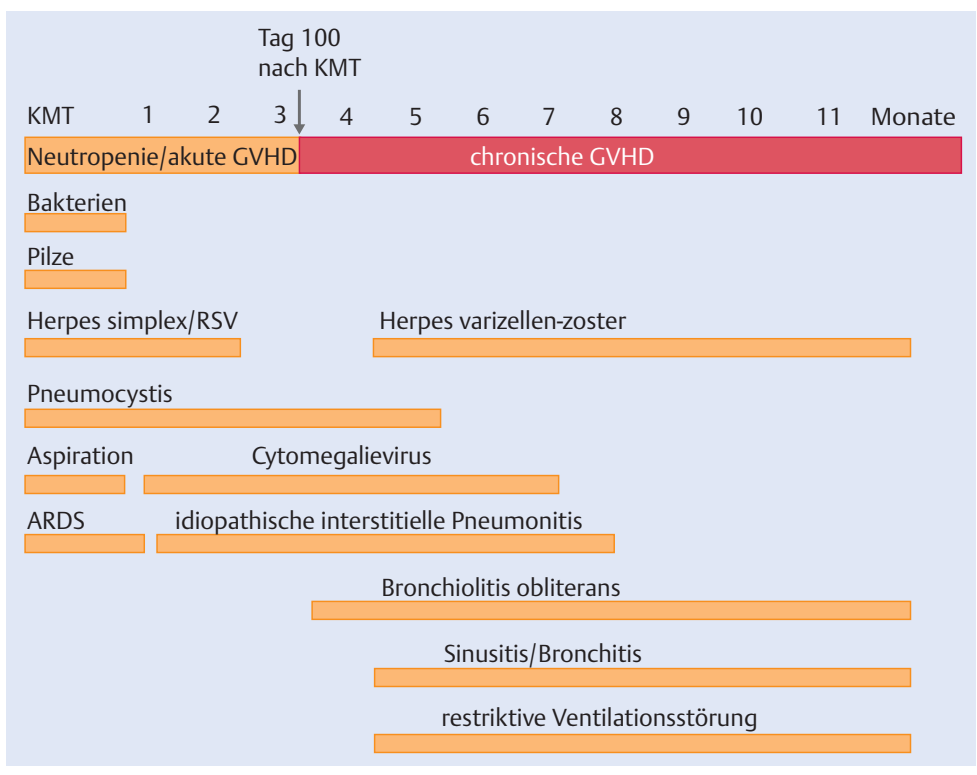

Abb. 7 Pulmonale Komplikationen während und nach der UMT und deren häufigste Ätiologie.
Zumindest in den ersten 30 Tagen nach der KMT sind regelmäßige, 2-mal pro Woche durchgeführte Screeeninguntersuchungen auf humanpathogene Viren mit quantitativer PCR notwendig. Hierbei ist es wichtig, den Verlauf der Kopienanzahl von CMV, Adenoviren, RSV, HHV6, EBV, Polyoma BK, Influenza und Parainfluenza-Viren zu beachten. Eine plötzliche Virämie sollte auch bei noch asyptomatischen Patienten zu einer medikamentösen Therapie Anlass geben [2]. Pulmonale Komplikationen nach KMT und ihr zeitlicher Bezug zur Transplantation sind in (Abb. 7) zusammengefasst.

CMV-Pneumonie. Die CMV-Pneumonie ist eine gefürchtete Organmanifestation einer endogenen CMVReaktivierung. Insbesondere die Kombination eines CMV-negativen Stammzellspenders mit einem CMVpositiven Empfänger ist eine Höchstrisikokonstellation. Aber auch bei einem CMV-positiven Spender kann der Empfänger eine schwere CMV-Reaktivierung erleiden.

schlichtweg nur „großes Glück“ gehabt haben, bleibt offen.

Naturgemäß fehlen geprüfte größere Untersuchungen oder klinische Studien zur optimalen Lokaltherapie eines Paravasats im Zusammenhang mit der intravenösen Zytostatikatherapie. Die kinderonkologische Fachgesellschaft hat jedoch einige Empfehlungen erarbeitet, die im Internet unter der URL www.kinderkrebsinfo.de/e1676/e1786/index_ger.html einsehbar sind.

\section{Komplikationen nach allogener Knochenmarktransplantation}

Die allogene Knochenmarktransplantation (KMT) ist für viele Kinder mit schwerem angeborenem Immunoder Stoffwechseldefekt, angeborenem oder erworbenem Knochenmarkversagen oder Hochrisikoleukämien die einzige kurative Therapie. Das Verfahren unterscheidet sich durch das immunologische Therapieprinzip grundsätzlich von autologen zellulären Therapieverfahren. Chancen und Risiken bei der Transplantation eines „neuen Immunsystems“ liegen dabei eng beisammen.

Der erfolgreiche Umgang mit den vielfältigen Komplikationen, die im Zusammenhang mit einer allogenen KMT auftreten können, erfordert auch zahlreiche Bausteine jenseits der pädiatrischen Onkologie und Intensivmedizin [1]. filtrate legen den Verdacht nahe. Ein gleichzeitiger Anstieg von CMV-Genomäquivalenten im peripheren Blut ist praktisch beweisend. Zur Therapie setzt man Ganciclovir ein, was auch bei klinisch noch asymptomatischer Virämie indiziert ist. Die Dosierung beträgt $2 \times 5 \mathrm{mg} / \mathrm{kg} \mathrm{KG} /$ Woche. Der Therapiekontrolle dient die PCR-Kontrolle der Viruslast. Nebenwirkungen sind eine toxische Wirkung auf das Knochenmark, Durchfall, Myopathie, Übelkeit und Erbrechen.

Wegen der stark myelosuppressiven Wirkung, die gerade bei Patienten nach der KMT problematisch ist, kommen auch Dosismodifikationen der Ganciclovirtherapie infrage. Bei ausbleibendem Therapieerfolg (Abfall der CMV-Viruslast, ganciclovirresistente CMVVarianten) sollte auf das Alternativpräparat Forscarnet ( $3 \times 60 \mathrm{mg} / \mathrm{kg} \mathrm{KG} / \mathrm{d})$ oder auf Cidofovir $(1 \times 5 \mathrm{mg} / \mathrm{kg} \mathrm{KG} /$ Woche) umgestellt werden. Auch hierfür sind alternative Therapieschemata $(3 \times 1 \mathrm{mg} / \mathrm{kg} \mathrm{KG} /$ Woche $)$ verwendet worden.

Der adoptive Transfer von CMV-spezifischen T-Lymphozyten, die aus dem peripheren Blut des ursprünglichen Stammzellspenders durch Spezialverfahren selektioniert werden, ist eine sehr elegante Methode zur Behandlung der CMV-Reaktivierung mit und ohne Organmanifestation. Sie ist aber nur an wenigen Zentren durchführbar und selbstverständlich nur dann in Betracht zu ziehen, wenn der Spender auch CMV-positiv ist.
Hypoxämie, Fieber, Husten und diffuse interstitielle In- 
Die Kombination von CMV-negativem Empfänger und CMV-negativem Spender ist in modernen KMT-Zentren eher unkritisch, da die CMV-Erstinfektion eines CMV-negativen Patienten durch Bluttransfusionen während der Transplantation der Vergangenheit angehören sollte.

Adenovirus, RSV, Parainfluenza, Influenza. Die Infektionen mit Adenoviren, RSV, Parainfluenza- und Influenzaviren treten im 2. und 3. Monat nach der Transplantation auf. Sie sind sehr ernst zu nehmen. Adenovirusinfektionen behandelt man ebenfalls mit dem sich allerdings noch im experimentellen Stadium befindlichen adoptiven Transfer adenovirusspezifischer TZellen [3].

HSV, VZV und HHV6B. Die HSV- und VZV-Reaktivierung ist Dank der Aciclovirprophylaxe seltener geworden. HHV6B ist ein häufiger Erreger einer Enzephalitis nach allogener KMT. Daneben sind Pneumonien und Knochenmarkaplasien beschrieben [4,5].

\section{Postransplantations-lymphoproliferative Erkran-} kung (PTLD). Die PTLD kann in der Folge aller Organtransplantationen auftreten. Da die immunsuppressive Medikation nach KMT nur passager und nicht - wie bei den organtransplantierten Patienten - lebenslang verabreicht werden muss, scheinen stammzelltransplantierte Kinder insgesamt weniger gefährdet zu sein als Kinder nach einer Herz- oder Nierentransplantation. Ebenso wie bei den CMV-Spender-/Empfängerkonstellationen gibt es auch für EBV Risikokonstellationen. Die PTLD entwickelt sich aus den Spender-B-Zellen. Es kommt zu einer unkontrollierten Proliferation von EBV in den transferierten B-Zellen, die schließlich auch in eine monoklonale Proliferation im Sinne eines B-NHL übergehen kann. Diagnostisch ist ebenfalls wieder die quantitative EBV-PCR wegweisend.

Die Therapie besteht - sofern klinisch möglich - in der Reduktion der Immunsuppression (Ciclosporindosis reduzieren), der Gabe des monoklonalen Antikörpers Rituximab (375 mg/m²) oder im adoptiven Transfer von EBV-spezifischen T-Zellen des Spenders. Der Therapieerfolg sollte sich auch an der Abnahme der EBV-Genomäquivalente um mindestens 1 log-Stufe orientieren. Die Substitution von Immunglobulinen ist hingegen ohne Nutzen.

\section{Transplantat-gegen-Wirt-Reaktion (Graft versus-}

Host-Disease, GvHD). Bei der GvHD handelt es sich um eine schwerwiegende Immunreaktion, bei der reife alloreaktive T-Lymphozyten des Spendertransplantats gegen Antigene auf dem Empfängergewebe reagieren. Man unterscheidet eine akute und eine chronische GvHD (> 100 Tage nach KMT). Sie geht mit einer ausgeprägten intrinsischen und medikamentös induzierten Immundefizienz einher, was das Risiko für schwerste Infektionen deutlich erhöht.

Die schweregradabhängige komplexe Therapie der akuten und chronischen GvHD kann an dieser Stelle nicht weiter erörtert werden; sie ist auch nur in seltenen Ausnahmefällen intensivmedizinisch relevant.

Eine ausgeprägte chronische GvHD hat auch heute noch eine schlechte Prognose. Die Kinder sind stark gefährdet, an einer unbehandelbaren septischen Komplikation zu versterben - unter Umständen liegt die eigentliche KMT dann schon mehrere Jahre zurück.

Lebervenenverschlusskrankheit. Die Lebervenenverschlusskrankheit („veno-occulssive disease“, VOD, auch sinusoidales Obstruktionssyndrom genannt) ist besonders nach allogener KMT gefürchtet, da sie mit einer hohen Letalität einhergeht ( $30 \%$ und mehr). Tritt die VOD im Rahmen einer autologen Stammzelltransplantation (Medulloblastom, Ewing-Sarkom) oder einer konventionellen chemotherapeutischen Behandlung (z.B. bei einem rechtsseitigen Wilms-Tumor) auf, ist die Prognose günstiger.

Durch die konditionierende Chemotherapie im Rahmen der KMT kommt es zur Schädigung des Gefäßendothels, was einen reduzierten venösen hepatischen Blutfluss bedingt. Morphologisch handelt es sich um einen konzentrischen, nicht thombotisch bedingten Verschluss terminaler und sublobulärer intraheptischer Venen. Es entwickelt sich eine postsinusoidale portale Hypertension mit Aszitesbildung. Die Reduktion der renalen Natriumausscheidung verstärkt die Flüssigkeitsretention mit oft erheblicher Gewichtszunahme. Es kommt zu einer meist schmerzhaften Hepatomegalie. Laborchemisch findet man typischerweise einen Bilirubinanstieg und eine oft foudryante Verschlechterung der Leberfunktionsparameter. Dopplersonografisch ist der portale Fluss vermindert, die oft beschriebene „komplette Flussumkehr“ in der Portalvene kann jedoch nicht immer beobachtet werden. Die Lebervenen sind hingegen schmal und zeigen ein normales Flussmuster.

Die Therapie der VOD ist schwierig, da sich niedrigdosiertes Heparin, AT III, Glutathion, Pentoxyphyllin oder Vitamin E als unwirksam erwiesen haben. 
Infobox 3

Defibrotide-Therapie bei Lebervenenverschlusskrankheit

- Beginn mit 10 mg/kg KG/d

- bei guter Verträglichkeit täglich um 10 mg/kg KG steigern bis maximal $60 \mathrm{mg} / \mathrm{kg} \mathrm{KG}$

- Tagesdosis auf 4 Einzelgaben verteilen

- wegen der Blutdruckwirksamkeit jede Einzeldosis als Infusion über $2 \mathrm{~h}$ geben

- keine gleichzeitige Gabe von Defibrotide und Heparin (Blutungskomplikationen)

Die einzige spezifische Therapie der VOD besteht in der Gabe von Defibrotide (Prociclide), welches über die internationale Apotheke von der Firma Crinos aus Italien beziehbar ist. Dabei handelt es sich um ein relativ schlecht definiertes Gemisch aus Polydesoxyribonukleotiden, das aus der Mucosa von Schweinelungen hergestellt wird. Der genaue Wirkmechanismus ist ungeklärt. Zur Dosierung s. Infobox 3.
Defibrotide ist ein Adenosinrezeptoragonist, moduliert die Thrombozytenaktivität und stimuliert die endogene Fibrinolyse über t-PA. Aufgrund der kurzen Halbwertszeit entfaltet es aber nur eine geringe systemische anitkoagulatorische Aktivität. Dennoch wurden im eigenen Krankengut schwere Blutungskomplikationen beobachtet.

Keine gleichzeitige Gabe von Defibrotide und Heparin wegen der möglichen Verstärkung von Blutungskomplikationen.

Weitere Nebenwirkungen umfassen insbesondere ein weites Spektrum von allergischen Reaktionen, Blutdruckabfall (Gefäßdilatation) sowie Erbrechen und Übelkeit.

Die Wirkung einer prophylaktischen Defibrotidegabe zur Vermeidung einer VOD in pädiatrischen KMT-Zentren wird derzeit im Rahmen einer prospektiven randomisierten klinischen Studie geprüft.

\section{Kernaussagen}

- Die komplexe, meist hochwirksame Behandlung von Leukämie und Tumorerkrankungen im Kindesalter hat die prinzipielle Chance auf Dauerheilung entscheidend verbessert. Die Therapie der Nebenwirkungen der Chemo- und Immuntherapie ist dafür von zentraler Bedeutung.

- Auch bei einer nur minimalen Atemstörung kann ein Mediastinaltumor innerhalb von nur wenigen Stunden eine schwere respiratorische Insuffizienz verursachen. Vor der Biopsie der mediastinalen Raumforderung in Allgemeinnarkose ist die Möglichkeit zu prüfen, durch weniger invasive Maßnahmen die Tumordiagnose zu stellen. Eine präoperative empirische Therapie für 48-72 h mit Kortikosteroiden und Cyclophosphamid ist zur Verkleinerung eines kritisch großen Mediastinaltumors sicherer als eine unverzügliche Biopsie.

- Die Rückenmarkkompression durch eine solide Raumforderung bedarf zur Vermeidung einer irreversiblen Querschnittsymptomatik einer raschen Intervention. Die Dauer der Kompression ist für die Restitution der nervalen Funktion entscheidend.

- Hyperleukozytose und Tumorlysesyndrom drohen insbesondere bei akuter myeloischer Leukämie, akuter lymphoblastischer Leukämie vom T-Zell-Typ und bei Säuglingsleukämie. Ein Tumorlysesyndrom kann durch eine Hyperkaliämie lebensbedrohliche Rhythmusstörungen verursachen.

- Fieber ist bei einer Neutropenie nach Chemotherapie oft das einzige Symptom einer beginnenden, schweren Allgemeininfektion. Daher ist zwingend eine frühzeitige empirische antibiotische Therapie erforderlich, auch wenn bei der Mehrzahl der Kinder die Ursache des Fiebers unklar bleibt und keine Keime nachgewiesen werden.

- Das theoretische und praktische Beherrschen möglicher Komplikationen einer hochdosierten Methotrexattherapie ist für kinderonkologische Behandlungszentren unerlässlich. Zur Behandlung der schweren MTX-Intoxikation steht das rekombinante Enzym Glucarpidase (Carboxypeptidase) zur Verfügung.
- Bei der allogenen Knochenmarktransplantation ist das regelmäßige Screening auf CMV, Adenoviren, RSV, HHV6, EBV, Polyoma BK-Virus, ggf. auch auf Influenza- und Parainfluenza-Virus notwendig. Auch bei asymptomatischen Patienten ist ein molekularbiologischer Nachweis von Virusgenom im peripheren Blut eine Therapieindikation. Die derzeitigen antiviralen Medikamente sind oft unzureichend wirksam und haben zahlreiche Nebenwirkungen. Der adoptive Transfer virusspezifischer T-Lymphozyten, die aus dem Blut des Stammzellspenders isoliert werden müssen, ist künftig die vielversprechendste Therapieoption. Virusspezifische T-Lymphozyten lassen sich derzeit zur Behandlung von CMV-, Adenovirusund EBV- Komplikationen erst in einigen wenigen Labors GMP-konform im klinischen Maßstab herstellen.

- Die Lebervenenverschlußerkrankung (VOD) nach allogener KMT hat eine hohe Letalität. Zur spezifischen Behandlung steht das Medikament Prociclide zur Verfügung. 
Neben Defibrotide wurde in jüngerer Zeit vereinzelt auch über den erfolgreichen Einsatz von aktiviertem humanem Protein C zur Behandlung der VOD bei Kindern berichtet [6]. Überraschenderweise hinterlässt die VOD - sofern die Kinder die schwere Erkrankung überleben - selten bleibende Leberschäden. Mit einer Resitutio ad integrum kann gerechnet werden [7].

Engraftment-Syndrom. Die unspezifische klinische Verschlechterung bei Kindern kurz vor oder im Zusammenhang mit dem Leukozytenanstieg im peripheren Blut (dem „take“) wird als Engraftment-Syndrom bezeichnet. Durch die begleitende Zytokinausschüttung der neu formierten Zellen kann es zu einem Schocksyndrom kommen.

Eine relativ plötzlich einsetzende Hypoxämie und Fieber um den Zeitraum eines vermuteten Leukozytenanstiegs weist auf ein Engraftment-Syndrom hin. Differenzialdiagnostisch ist eine peraktute GvHD abzugrenzen. Durch die mittlerweile praktisch flächendeckend eingeführten hochauflösenden Spender-EmpfängerGewebetypisierungen in der pädiatrischen KMT ist die perakute GvHD jedoch selten geworden. Die Therapie mit Steroiden (1 - $2 \mathrm{mg} / \mathrm{kg} \mathrm{KG}$ ) ist eine wirksame Maßnahme.

\section{Über den Autor}

\section{Arndt Borkhardt}

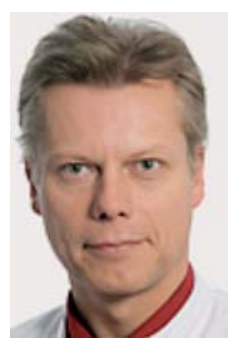

Jahrgang 1963. Prof. Dr. med. Studium der Medizin in Magdeburg. 1990 Approbation und Promotion. 1991 - 1997 Assistenzarzt an der Universitäts-Kinderklinik Gießen, Facharzt und ab 1998 Oberarzt in der pädiatrischen Intensivmedizin und Neonatologie in Gießen, 1999 auch in der Kinderonkologie als Oberarzt tätig.

Annerkennung in den Weiterbildungsschwerpunkten Neonatologie (1999), pädiatrische Intensivmedizin (2003) und Kinderonkologie (2004). 2003 Wechsel nach München und Rufannahme auf die C3-Professur der LMU München (Dr. v. Hauner'sches Kinderspital). 2006 Rufannahme auf den Lehrstuhl für Kinder-Onkologie, -Hämatologie und Klinische Immunologie der Heinrich-Heine-Universität Düsseldorf. Direktor der gleichnamigen Klinik.

\section{Korrespondenzadresse}

Prof. Dr. Arndt Borkhardt

Klinik für Kinder-Onkologie, -Hämatologie

und Klinische Immunologie

Moorenstr. 5

40225 Düsseldorf

Telefon: 0211/81-17680

Telefax: 0211/81-16707

E-mail: lesch@med.uni-duesseldorf.de

\section{Literatur}

1 Gadner H, Gaedicke G, Niemeyer C, Ritter J. Pädiatrische Hämatologie und Onkologie. Berlin, Heidelberg, New York: Springer, 2006

2 Boeckh $M$. The challenge of respiratory virus infections in hematopoietic cell transplant recipients. $\mathrm{Br}$ J Haematol 2008; 143: $455-467$

3 Feuchtinger T, Richard C, Joachim S et al. Clinical grade generation of hexon-specific $T$ cells for adoptive $T$-cell transfer as a treatment of adenovirus infection after allogeneic stem cell transplantation. J Immunother 2008; 31: $199-206$

4 Johnston RE, Geretti AM, Prentice HG et al. HHV-6-related secondary graft failure following allogeneic bone marrow transplantation. Br J Haematol 1999; 105: 1041 - 1043

5 Bosi A, Zazzi M, Amantini A, Cellerini M et al. Fatal herpesvirus 6 encephalitis after unrelated bone marrow transplant. Bone Marrow Transplant 1998; 22: 285- 288

6 Eber SW, Gungor T, Veldman A et al. Favorable response of pediatric stem cell recipients to human protein $C$ concentrate substitution for veno-occlusive disease. Pediatr Transplant 2007; 11: $49-57$

7 Corbacioglu S, Greil J, Peters C et al. Defibrotide in the treatment of children with veno-occlusive disease (VOD): a retrospective multicentre study demonstrates therapeutic efficacy upon early intervention. Bone Marrow Transplant 2004; 33: $189-195$

8 Bigham MT, Nowak JE, Wheeler DS. Therapeutic application of helium-oxygen and mechanical ventilation in a child with acute myelogenous leukemia and airway obstruction. Pediatr Emerg Care 2009; 25: 469-472 


\section{CME-Fragen}

\section{CME.thieme.de}

CME-Teilnahme

- Viel Erfolg bei Ihrer CME-Teilnahme unter http://cme.thieme.de

- Diese Fortbildungseinheit ist 12 Monate online für eine CME-Teilnahme verfügbar.

- Sollten Sie Fragen zur Online-Teilnahme haben, unter http://cme.thieme.de/hilfe finden Sie eine ausführliche Anleitung.

1

Welche Aussage im Zusammenhang mit einem großen Mediastinaltumor mit deutlichem Pleuraerguss, aber mäßiger Dyspnoe ist richtig?
A Der Patient ist zu intubieren und die histologische Diagnostik des Tumors unverzüglich anzustreben.

B Die Punktion des Pleuraergusses erlaubt die Diagnostik und bessert die respiratorische Symptomatik.

C Es handelt sich in der Regel um Keimzelltumoren, die durch die Tumormarker $\beta$-HCG und $\alpha$-Fetoprotein charakterisiert sind.

D Die Gabe von Steroiden ist auch bei respiratorischer Beeinträchtigung unbedingt zu vermeiden, um eine nachfolgende histologische Diagnostik nicht zu verhindern.

E Auf invasive Maßnahmen ist grundsätzlich zu verzichten. Es ist auf eine ausreichende Diurese und Flüssigkeitszufuhr zu achten.
Bei einem 8-jährigen, vorher gesunden Jungen mit plötzlicher Gangunsicherheit ergab die MRTBildgebung den Verdacht auf eine Rückenmarkkompression durch einen soliden Tumor. Weitere Raumforderungen werden nicht gefunden. Das Blutbild ist unauffällig. Was ist zu tun?
A Ein chirurgischer Eingriff mit einer Hemilaminektomie/Laminektomie muss unverzüglich durchgeführt werden. Es droht sonst die irreversible Querschnittlähmung.

B Der Patient ist lumbal zu punktieren, da die Wahrscheinlichkeit hoch ist, dass Tumorzellen im Punktat die Diagnose der Raumforderung ermöglichen.

C Das Kind hat wahrscheinlich trotz des normalen Blutbilds eine Leukämie und muss mit Steroiden behandelt werden.

D Es handelt sich meist um ein in die Formina intervetebralia eingewachsenes Neuroblastom, das praktisch nie zu einem kompletten Querschnitt führt. Die Chemotherapie kann ohne bioptische Sicherung eingeleitet werden.

E Solange die Blasenentleerung ungestört möglich ist, sollte die weitere apparative Diagnostik zum Tumorstaging komplettiert werden.
Bei einem 8-jährigen Kind mit chronisch myeloischer Leukämie beträgt die initiale Leukozytenzahl

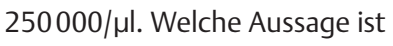
richtig?
A Eine Austauschtransfusion ist indiziert und sollte möglichst rasch durchgeführt werden.

B Wegen der besonderen Chemotherapiesensibilität pädiatrischer Leukämien ist vorsichtig mit der Steroidtherapie zu beginnen.

C Die Indikation zur Austauschtransfusion richtet sich nach dem klinischen Bild.

D Ein Tumorlysesyndrom ist bei dieser Erkrankung sehr wahrscheinlich, die Gabe rekombinanter Uratoxydase ist indiziert.

E Wegen der besonderen Blutungsgefahr ist auf die Asparaginasegabe zu verzichten, bis sich die Zellzahlen der Leukozyten normalisiert haben.
Worauf ist bei einem drohenden Tumorlysesyndrom besonders zu achten?
A auf die Entwicklung einer Hyperkaliämie
B Alkalisierung des Harns, um das Ausfallen von Xanthin und Harnsäure zu vermeiden
C Allopurinolzufuhr, da sie eine sichere und effektive Maßnahme zum Schutz einer Uratnephropathie ist
D Urin pH-Wert um 7 halten, um die Ausfällung von Kalziumphosphat zu vermeiden
E rechtzeitige Gabe von rekombinanter Uratoxydase zur enzymatischen Spaltung der Harnsäure 


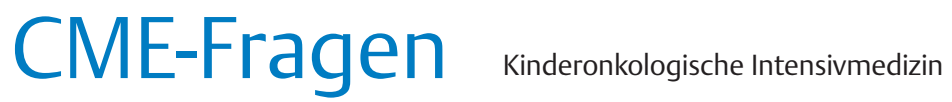

\section{5}

Welche Aussage trifft für

Methotrexat (MTX) zu?
A Ein Paravasat hat schwere Hautnekosen zur Folge. Deswegen sollte MTX immer über einen zentralvenösen Katheter verabreicht werden.

B eine MTX-Ausscheidungsstörung ist lebensbedrohlich und kann mit dem MTX-spaltenden Medikament Glucarpidase wirksam behandelt werden.

C MTX führt bei versehentlicher intrathekaler Gabe zu schweren, meist tödlichen Nebenwirkungen.

D Die MTX-Spiegelbestimmung wird durch Gabe des Antidots Leucovorin gestört.

E Die MTX-Therapie kann zu kardialen Rhythmusstörungen durch den Kalziumgehalt im Präparat führen.

6

Welche Aussage zur Sinusvenenthrombose bei Kindern unter der Therapie einer Leukämie ist falsch?

\section{7}

Welche Aussage ist richtig?

\section{8}

Welche Aussage trifft zu?
A soll mit niedermolekularem Heparin behandelt werden
B führt zur Erhöhung des intrakraniellen Drucks
C bei gesicherter Sinusvenenthrombose rasch eine Fibrinolysetherapie einleiten
D hat eine gute Prognose, da die Rate an Rekanalisierungen hoch ist
E tritt gehäuft bei Kindern unter der Therapie mit Asparaginase auf

A Die CMV-Pneumonie ist eine häufige, gefährliche Komplikation der autologen KMT.

B Die CMV-Pneumonie tritt besonders bei einem CMV-negativen Spender für einen CMV-positiven Empfänger auf.

C Die Therapie mit Ribavarin sollte frühzeitig auch bei asyptomatischen Patienten eingeleitet werden.

D Die CMV-Erstinfektion während der allogenen KMT verläuft häufig unbemerkt.

E Die Diagnostik mit PCR aus dem peripheren Blut kann nur in Verbindung mit typischen radiologischen Veränderungen für eine Therapieeinleitung genutzt werden.

A Die Lebervenenverschlusserkrankung (VOD) kommt bei einem linksseitigen Wilms-Tumor gehäuft vor.

B Das Auftreten einer VOD im Rahmen einer allogenen KMT führt praktisch immer zu bleibenden Leberschäden.

C Für die erfolgreiche Behandlung der VOD ist die frühzeitige Gabe von AT III entscheidend.

D Bei Behandlung der VOD mit Prociclide ist darauf zu achten, dass Prociclide nicht mit Heparin kombiniert eingesetzt wird.

E Eine sonografisch gesicherte Flussumkehr in den Lebervenen ist ein typisches Zeichen für eine VOD. 


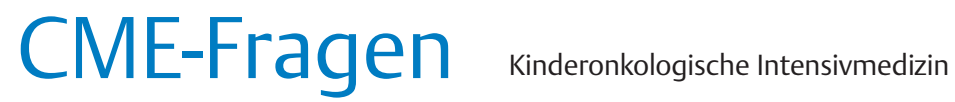

\section{9}

Wie kann eine ausgeprägte Posttransplantations-lymphoproliferative Erkrankung (PTLD) nach einer allogenen KMT wirksam behandelt werden?
A Ganciclovir
B Ribavirin
C Rituximab
D Aciclovir
E Verstärkung der Immunsuppression, z. B. Anhebung des Ciclosporin-A-Spiegels

10

Durch welche Besonderheit zeichnet sich eine akute myeloische Leukämie vom FAB-Subtyp M3 (Promyelozytenleukämie) aus?
A Kommt meist bei jungen Säuglingen vor.

B Geht auch bei moderat erhöhter Leukozytenzahl mit einer hohen Rate an schwerwiegenden Blutungskomplikationen einher.

C Rezidiviert auch nach Erreichen der Remission rasch und hat insgesamt eine schlechte Prognose.

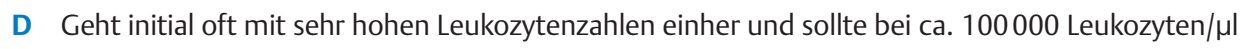
obligat mit einer Austauschtransfusion behandelt werden.

E Die Indikation zur Austauschtransfusion sollte sich primär am klinischen Bild und weniger an der absoluten Zahl der Blasten im peripheren Blut orientieren. 